\title{
Spin-dependent photophysics in polymers lightly doped with fullerene derivatives: Photoluminescence and electrically detected magnetic resonance
}

\author{
B. Zerai Tedlla, ${ }^{1}$ F. Zhu, ${ }^{2}$ M. Cox,${ }^{2}$ B. Koopmans, ${ }^{2}$ and E. Goovaerts ${ }^{1}$ \\ ${ }^{1}$ Department of Physics, University of Antwerp, Universiteitsplein 1, B-2610 Antwerpen, Belgium \\ ${ }^{2}$ Department of Applied Physics, Center for Nano Materials (CNM), Eindhoven University of Technology, \\ 5600 MB Eindhoven, The Netherlands \\ (Received 8 December 2014; revised manuscript received 18 January 2015; published 23 February 2015)
}

\begin{abstract}
Lightly fullerene-doped polymers are suitable composite systems to study spin-dependent bimolecular interactions among charge excitations due to their long lifetimes in these systems. These interactions can affect the photocurrent as well as the open-circuit voltage in an organic solar cell. Combining photoluminescence detected magnetic resonance (PLDMR) and electrically detected magnetic resonance (EDMR) spectroscopies we study films and devices of poly ( $p$-phenylene vinylene) polymers poly[2-methoxy-5-( $3^{\prime}, 7^{\prime}$-dimethyloctyloxy)1,4-phenylene vinylene] (MDMO-PPV) and superyellow PPV (SY-PPV) lightly doped with various fullerene derivatives [6,6]-phenyl $\mathrm{C}_{61}$ butyric acid methyl ester (PCBM), bis[60]PCBM (bis-PCBM), indene- $\mathrm{C}_{60}$ bisadduct (ICBA), and [6,6]-phenyl $\mathrm{C}_{71}$ butyric acid methyl ester (PC70BM). (i) We demonstrate strong fullerene triplet exciton (TE) production in SY-PPV:fullerene blends, whereas this is absent in MDMO-PPV:PCBM and only very weak in MDMO-PPV:ICBA. The low TE production in blends with MDMO-PPV is attributed to a weaker singlet-singlet energy-transfer coupling and an unfavorable triplet level alignment between the blend components. (ii) The fullerene TE spectra are analyzed on the basis of a single type of triplet excitation in PCBM, bis-PCBM, and ICBA, and two triplet species in PC70BM which are attributed to the $\alpha$ - and $\beta$-type isomers of the latter molecule. (iii) The sign change with increasing temperature of the $g \sim 2$ sharp central line in photo-EDMR, which is observed both in pristine SY-PPV and in blends with fullerene, is correlated to a transition from dominant TE-polaron annihilation to nongeminate polaron recombination processes.
\end{abstract}

DOI: 10.1103/PhysRevB.91.085309

PACS number(s): 72.25.Dc, 73.50.Gr, 76.70.Hb, 88.40.jr

\section{INTRODUCTION}

The presence of different charge excitations in polymer:fullerene solar cells with an impact on the photovoltaic processes at widely varying time scales [1-3] inevitably makes the photophysics complex and at the same time intriguing. Above band-gap photoexcitation in a polymer:fullerene solar cell results in singlet excitons (SEs) both in the polymer and in the fullerene components. Driven by the difference in energy between either the lowest unoccupied molecular orbitals or the highest occupied molecular orbitals of the polymer and fullerene component materials, SEs at the polymer:fullerene interface first relax to the charge-transfer (CT) state and then dissociate into separated polarons (Ps). Subsequently, the Ps are whisked away to the appropriate electrodes via the polymer or the fullerene routes.

However, during this course of action Ps or their precursors can be diverted into parasitic pathways that hamper photovoltaic performance. For example, SEs can be lost via both radiative and nonradiative recombination to the ground state and by intersystem crossing (ISC) to triplet excitons (TEs) [4]. Separated Ps at the interface can also get trapped, particularly at defect sites in each material component as well as in the molecularly mixed polymer:fullerene zone where there are no meaningful quasicontinuous polymer or fullerene percolation paths for long-range $P$ transport [5-7]. Both geminate and nongeminate recombinations of separated Ps can also occur before they are extracted at the appropriate electrodes $[8,9]$.

Thus, in operating solar cell SE, TE, CT pairs, trapped and/or free Ps, bipolarons (BPs) (bound Ps of the same polarity), and possibly more exotic species, such as trions (weakly bound TE-P) may coexist [10,11]. In optimized bulk heterojunction polymer:fullerene solar cells, SEs have extremely short-lived lifetimes ( $\tau \sim 30-100 \mathrm{fs}$ ) [12] whereas TEs and Ps have respective lifetimes at room temperature in the $\sim 100$-ns range and in the microsecond to millisecond range, respectively $[1,13]$. Magnetic-field effect spectroscopy, such as organic magnetoresistance (OMAR) identifies the interaction between these charge excitations based on their contribution to the conductivity (current) in the organic solar cell devices when an applied magnetic field is swept beyond the hyperfine field ( $\sim$ tens of milliteslas) arising from the protons of the hydrogen atoms [14]. Charge interaction models, such as P-pair recombination [15], BP formation [16], TE-P annihilation [17], trion (weakly bound TE and P) formation [18], and TE-TE annihilation [19] have been put forward for the interpretation of the experimental observations. Magnetic-resonance effects via change in photoluminescence (PL) and electroluminescence (EL) have also been employed to identify different charge interactions in organic photovoltaic devices and in both fluorescent and phosphorescent organic light-emitting diodes (OLEDs) [20-22].

Magnetic-resonance spectroscopies are probably the most selective and direct methods for identification of some of these charge excitations via their unique magnetic-resonance signatures, their $g$ values, and in the case of TEs their zero-field splitting (ZFS) parameters [23]. In particular, the probability of spin-dependent interactions, such as TE-P, P-P, TE-TE, and of resulting reactions can be modified due to the redistribution of spins among the different magnetic (Zeeman) sublevels at magnetic-resonance conditions. Therefore, this can also have an impact on photon absorption, PL, EL, and 
(photo)current in operating organic solar cells, or OLEDs at magnetic resonance. By detecting the change in these macroscopic observables under resonance conditions and with the aid of the unique magnetic-resonance features, both the identity of the charge excitations and their spin-dependent interactions can systematically be investigated. In this respect, lightly fullerene-doped polymers are suitable systems due to the presence of different charge excitations in either or both blend components on a time scale sufficiently long for their selective detection via magnetic resonance.

In this paper we combine parallel photoluminescence detected magnetic resonance (PLDMR) and electrically detected magnetic resonance under photoexcitation (photo-EDMR) to investigate photophysical processes in lightly fullerene-doped poly ( $p$-phenylene vinylene) (PPV) polymer films and devices. We further determine the spin-Hamiltonian parameters of the TEs in a series of fullerene derivatives. Finally, we elucidate spin-dependent interactions based on their impact on photocurrent and PL intensity which points to a combination of TE-P annihilation, dominant at low temperatures, and recombination of distant $\mathrm{P}$ pairs.

\section{EXPERIMENTAL METHODS}

Polymer:fullerene films and devices were prepared from either of the polymers poly[2-methoxy-5-(3',7'dimethyloctyloxy)-1,4-phenylenevinylene] (MDMO-PPV) and "superyellow" PPV (SY-PPV) blended with one of the fullerene derivatives [6,6]-phenyl $\mathrm{C}_{61}$ butyric acid methyl ester (PCBM), bis[60]PCBM (bis-PCBM), indene- $\mathrm{C}_{60}$ bisadduct (ICBA), and [6,6]-phenyl $\mathrm{C}_{71}$ butyric acid methyl ester (PC70BM) acceptors. MDMO-PPV and SY-PPV were purchased from Sigma-Aldrich and Merck, respectively, and the four fullerene derivatives PCBM, PC70BM, ICBA, and PC70BM were purchased from Solenne. All materials are used as delivered. Solutions in chlorobenzene of the polymers and of the fullerene molecules $(6 \mathrm{mg} / \mathrm{ml}$ each) were prepared separately and stirred overnight.

For EDMR investigations, arrays of 12 devices with individual active areas of $5 \times 2 \mathrm{~mm}^{2}$ were deposited on patterned indium tin oxide- (ITO-) coated glass substrates $\left(3 \times 3 \mathrm{~cm}^{2}\right)$. The latter were pre-engraved from the backside to permit easy breaking between the devices after fabrication. The EDMR devices have the structure ITO/poly $(3,4-$ ethylenedioxythiophene-poly(styrene sulfonate) (30 nm)/ active-layer $(90-100 \mathrm{~nm}) / \mathrm{LiF}(1 \mathrm{~nm}) / \mathrm{Al}(80 \mathrm{~nm})$. Electrical contact is maintained by connecting two external wires embedded on a holder to the ITO and LiF/Al electrodes of the device using silver paste with the front side of the device accessible for illumination. The PLDMR films were either broken from metal-free parts of the EDMR devices or spin coated on ITO-coated glass substrates in cases where only PLDMR experiments were performed. Both EDMR and PLDMR experiments were performed on a cw X-band EPR spectrometer (Bruker ESP300E, 9.44 GHz) in a rectangular TE102 resonator with optical access. The instrument is equipped with a helium flow cryostat (Oxford Instruments, Inc.) allowing temperature variation between $T=2.4$ and $300 \mathrm{~K}$.

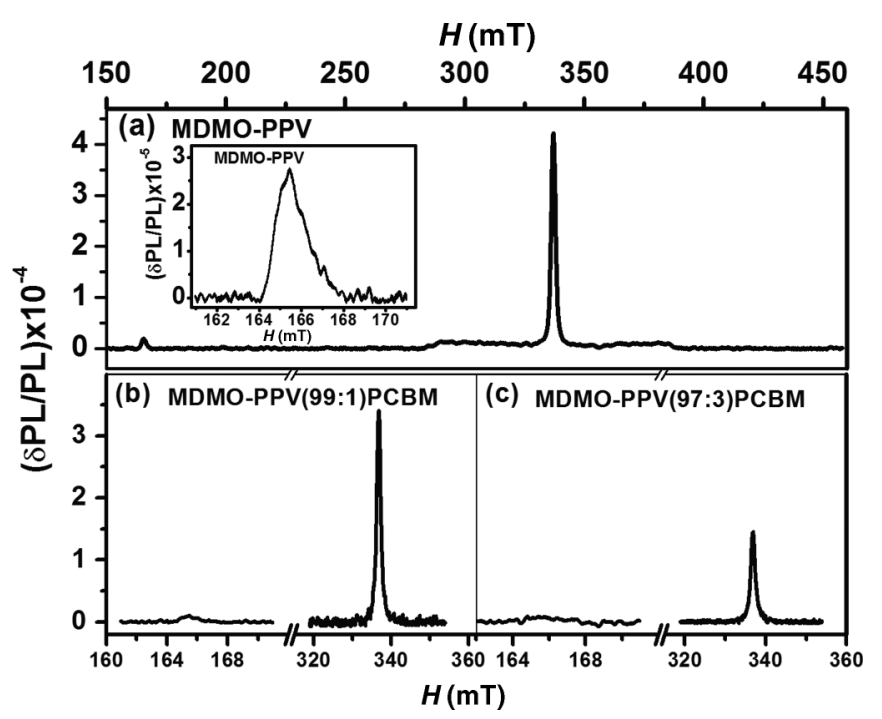

FIG. 1. (a) PLDMR spectra (modulus) of pristine MDMO-PPV. The inset zooms in onto the asymmetric half-field spectrum at $H \cong 165.4 \mathrm{mT}$. The PLDMR spectra of (b) MDMO-PPV(99:1)PCBM and (c) MDMO-PPV(97:3)PCBM $(T=3.2 \mathrm{~K})$. Both pristine and blend films are photoexcited at $2.54 \mathrm{eV}(\lambda=488 \mathrm{~nm})$ close to the absorption peak of the MDMO-PPV polymer.

In PLDMR measurements samples were placed close to the tip of an optical fiber that is embedded inside a dedicated sample holder. The PL from the samples passing through the optical port of the resonator is filtered and focused on a diode photodetector connected to a lock-in amplifier. The reported PLDMR signal is the relative resonant change in PL signal. In the EDMR measurements, the devices were connected in series with an external load resistor and a Keithley 2004 source meter. The voltage across the load resistor is then fed into a lock-in amplifier. The EDMR signal is the relative resonant change in photocurrent across the device. All EDMR measurements were performed at zero external bias under laser excitation of the device through the optical port of the resonator.

During the PLDMR and EDMR experiments the microwaves (MWs) are ON/OFF modulated at a frequency of $405 \mathrm{~Hz}$. The in-phase and quadrature signals are recorded in parallel, and eventually the modulus signal is calculated.

Optical excitation of both films and devices was achieved using $\mathrm{Ar}^{+}$laser beams of wavelength $\lambda=457 \mathrm{~nm}$ for SY:PPV:fullerene samples and $\lambda=488 \mathrm{~nm}$ for MDMO-PPV:fullerene samples, close to the absorption maxima of the polymers.

\section{RESULTS AND DISCUSSION}

\section{A. TE formation in lightly fullerene-doped polymers}

Figures 1 and 2 show the modulus PLDMR spectrum in lightly PCBM- and ICBA-doped MDMO-PPV blend films with varying fullerene fractions. For comparison, the PLDMR signal in a pristine MDMO-PPV film is also shown in 


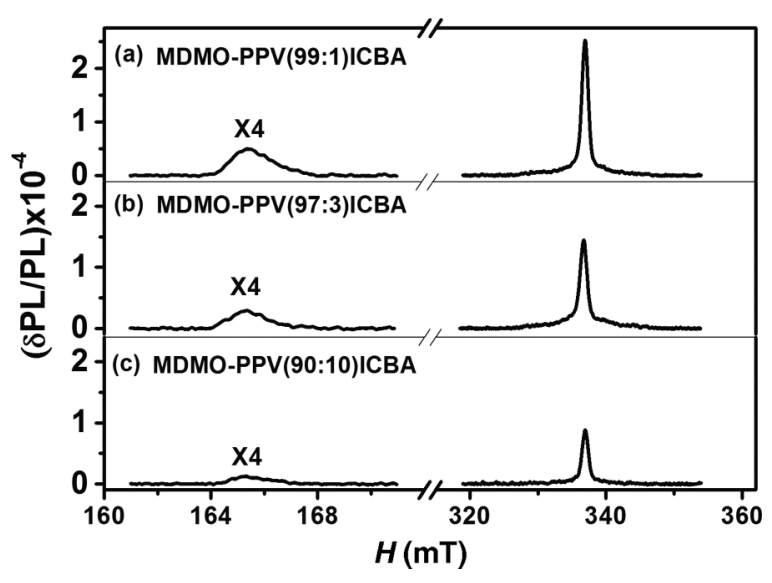

FIG. 2. PLDMR spectra (modulus) of (a) MDMOPPV(99:1)ICBA, (b) MDMO-PPV(97:3)ICBA, and (c) MDMO-PPV(90:10)ICBA blend films ( $T=3.2 \mathrm{~K})$. The half-field PLDMR triplet signature of MDMO-PPV is still visible in a blend film with $10 \%$ ICBA. The blend films are photoexcited at $2.54 \mathrm{eV}(\lambda=488 \mathrm{~nm})$.

Fig. 1(a). The PLDMR signal of the pristine MDMO-PPV film comprises: (i) a sharp $g \sim 2$ central peak that was previously attributed to recombination of distant polarons, (ii) a weak and broad full-field triplet spectrum of width $\sim 100 \mathrm{mT}$ centered around $g \sim 2$ and related to the MW-induced transitions with $\left|\Delta m_{\mathrm{s}}\right|=1$ between spin states of the polymer TE, and (iii) a half-field line at $\sim 165.4 \mathrm{mT}$ corresponding to the so-called forbidden transition between the spin states $m_{\mathrm{s}}= \pm 1$ $[21,22,24-26]$. Due to weak ISC in PPVs [27] and the wide spread of the full-field triplet spectrum, the presence of TEs in the MDMO-PPV component is most sensitively monitored via the half-field transition. The position $\left(\sim 165.4 \mathrm{mT}, g_{\text {eff }} \cong 4.1\right)$ and the asymmetry of the half-field line [shown in the inset of Fig. 1(a)] arise from second-order contributions of the relatively large ZFS (also evident from the width of the full-field spectrum).

In MDMO-PPV:PCBM blend films the intensity of this half-field polymer TE spectrum weakens when the PCBM concentration is increased and vanishes at a PCBM concentration of $10 \%$ (not shown here) consistent with a previous report by Scharber et al. [28]. In MDMO-PPV:ICBA blend films the half-field signature in the polymer is still clearly visible at a $10 \%$ compositional fraction of ICBA, despite strong reduction in intensity. In addition, a very weak but narrower (compared to the powder spectrum of MDMO-PPV) full-field triplet spectrum is appearing around the $g \sim 2$ sharp central peak. This signal originates from TEs in ICBA, however no half-field PLDMR spectrum corresponding to the ICBA is detected. This may be due to the extremely weak TE population in ICBA molecules in these blends.

Figure 3 shows the drastically different photo-EDMR spectra of the blends of SY-PPV with three fullerenes which exhibit strong fullerene triplet signals resulting from efficient energy transfer (ET) followed by direct ISC on the fullerenes as we discussed previously [29]. These are observed both in the full-field and in the half-field regions and in the PLDMR

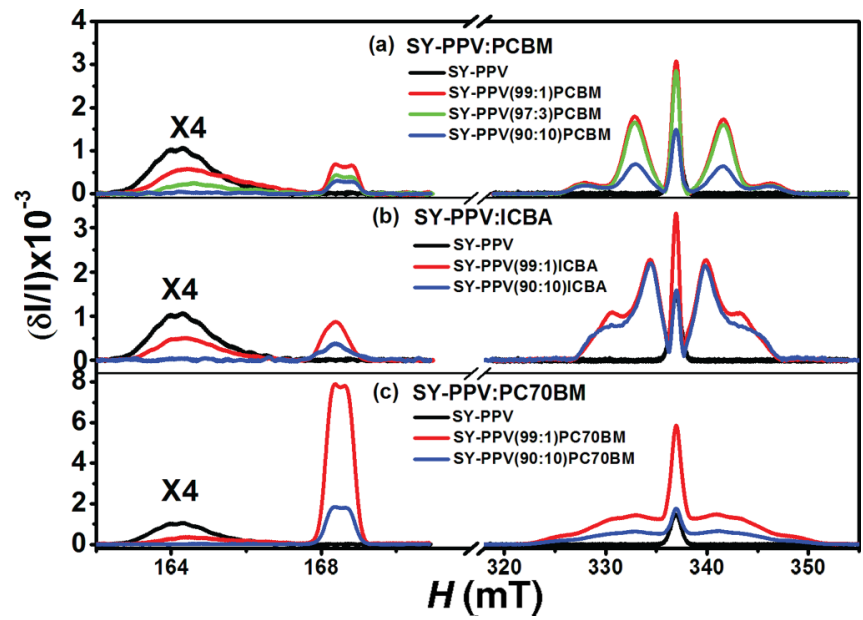

FIG. 3. (Color online) The photo-EDMR spectra (modulus) of (a) SY-PPV:PCBM, (b) SY-PPV:ICBA, and (c) SY-PPV:PC70BM blend devices with different fullerene concentrations $(T=3.2 \mathrm{~K})$. Devices are photoexcited at $2.71 \mathrm{eV}(\lambda=457 \mathrm{~nm})$, near the absorption peak of the SY-PPV polymer. The full-field and the corresponding ( $\left.H \cong 168.5 \mathrm{mT}, g_{\text {eff }} \sim 4.0\right)$ half-field spectra of fullerene TEs are clearly visible. The half-field TE spectra of the SY-PPV blend components are also shown at $H \cong 164.5 \mathrm{mT}\left(g_{\text {eff }} \sim 4.1\right)$.

spectra (see Fig. 4). Whereas the full-field spectrum of the polymer TE is hard to detect in these blends, the half-field TE signatures of both blend material components are easily observed and distinguished because of their different positions and linewidths: The narrower fullerene peak appears at nearly exactly half field ( $g_{\text {eff }} \cong 4.0$ ) because of the smaller ZFS (see also Sec. III B). Similar spectra with TE signatures of both blend components are also detected in SY-PPV:bis-PCBM blend films and/or devices (discussed below). The relative strength of the full-field spectrum of the fullerene TEs results partly from the narrower field spread of their powder spectrum $(\sim 1 / 5$ of that in PPVs).

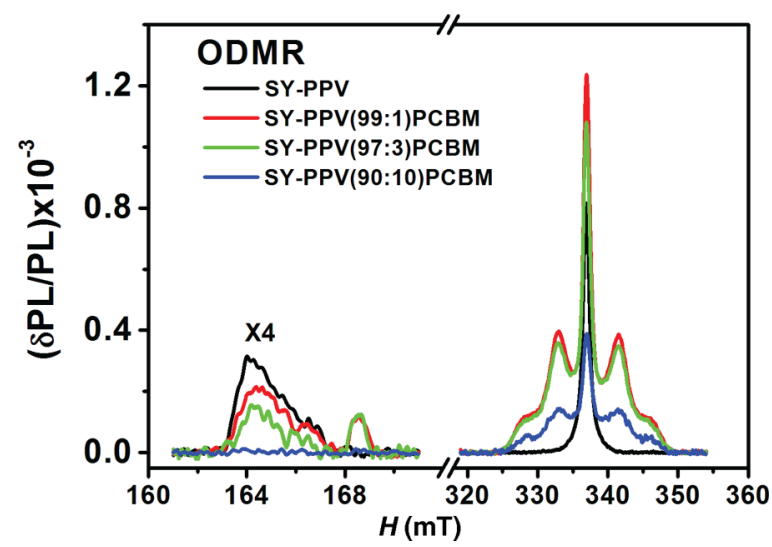

FIG. 4. (Color online) The PLDMR spectra (modulus) of SYPPV:PCBM blend films $(T=3.2 \mathrm{~K})$. Films are photoexcited at $2.71 \mathrm{eV}(\lambda=457 \mathrm{~nm})$. The full-field and the corresponding half-field $\left(H \cong 168.5 \mathrm{mT}, g_{\text {eff }} \sim 4.0\right)$ spectra of fullerene molecules are clearly visible. Similar PLDMR spectra are observed for SY-PPV:ICBA and SY-PPV:PC70BM blend films (not shown here). 


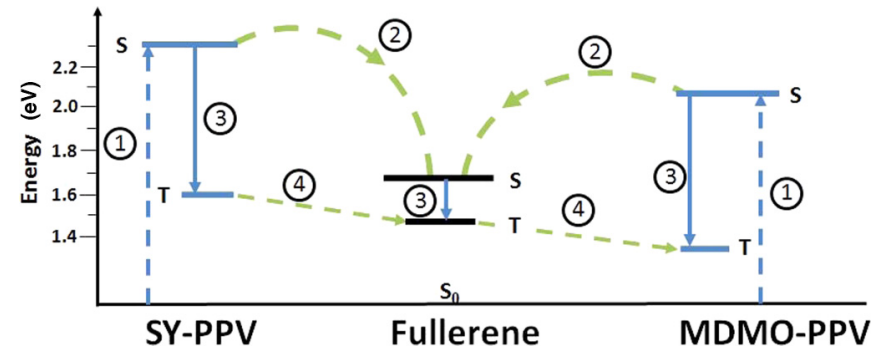

FIG. 5. (Color online) A schematic of triplet formation pathways in lightly fullerene-doped SY-PPV and MDMO-PPV polymers, (1) photoexcitation of a blend film resulting in generation of SEs, (2) SE-SE ET from the polymers to enclosed fullerene molecules, (3) ISC in each component material, and (4) triplet-triplet ET that populates the lower-lying triplet states.

A schematic of the possible photophysical pathways leading to TE formation in the blend films is shown in Fig. 5. Photoexcitation in the polymer:fullerene blend films generates SEs in the polymer components. Direct photon absorption by the fullerene molecules is negligible due to a combination of their low concentration in the blend films and their intrinsic weak absorption capabilities. Some of the SEs in the polymers can reach the fullerene by ET, others either recombine to the ground states or convert to TEs in the polymer by ISC. Likewise, energy-transferred SEs in fullerene can also recombine to the ground state or convert to TEs by direct ISC. At such low fullerene weight fractions in the blend films, CT formation at the polymer:fullerene molecular contact sites is very limited, and subsequently charge dissociation probabilities are very low.

The occurrence of specific Dexter-type triplet-triplet ET processes depends on the relative energies of the triplet states in each of the compounds of the blend (see Fig. 5). The triplet level energy $\left(E_{\mathrm{T}}\right)$, relative to the ground state in SY-PPV, MDMO-PPV, and the fullerene molecules is reported to be $1.6 \mathrm{eV}$ [30], $1.4 \mathrm{eV}$ [31], and $1.5 \mathrm{eV}$ [32], respectively. Therefore, $E_{\mathrm{T}}$ in the fullerene components is higher than that in MDMO-PPV, and in these blends TEs in the fullerene may decay to the low-lying triplet state of the polymer. Since the steady-state population of fullerene TEs is mainly dependent on the singlet-singlet ET from the polymer to the fullerene (due to the unfavorable $E_{\mathrm{T}}$ between the blend components for TE-TE energy transfer from the polymer to the fullerene), the absence or very limited population of fullerene TEs in the MDMO-PPV:fullerene blend can be attributed to a weak singlet-singlet ET coupling between MDMO-PPV and fullerene molecules. In SY-PPV:fullerene blends the $E_{\mathrm{T}}$ of the fullerene component is lower in energy than that of the polymer, and the fullerene TE is populated both by the ISC from the corresponding singlet and, to a lesser extent (due to the small ISC yield in SY-PPV), by the triplet-triplet ET from the polymer. Therefore, this favorable triplet energy alignment in SY-PPV:fullerene combined with a strong singlet-singlet ET from the polymer to the fullerene component in this blend leads to a strong population of fullerene triplets starting from photoexcited SEs in the polymer. This is consistent with our observation of very strong full-field fullerene TE signatures

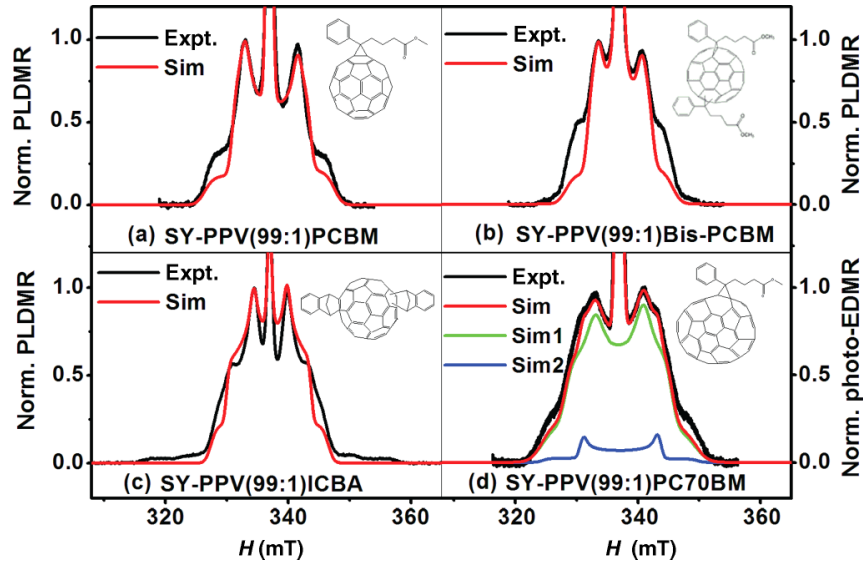

FIG. 6. (Color online) The in-phase PLDMR [photo-EDMR in (d)] spectra and the corresponding EPR simulation for (99:1) blends of SY-PPV with (a) PCBM, (b) bis-PCBM, (c) ICBA, and (d) PC70BM. The EPR simulation of the photo-EDMR spectrum of SY-PPV(99:1)PC70BM device is a linear combination of two triplet spectra, (Sim1 and Sim2), corresponding to the $\alpha$ - and $\beta$-type isomers, respectively, with 85:15 composition.

in PLDMR as well as photo-EDMR spectra of these blend systems.

\section{B. Zero-field splitting parameters of fullerene and polymer triplet excitons}

Taking advantage of the strong fullerene TE production in the SY-PPV:fullerene blend, we determine the spinHamiltonian parameters, in particular, the ZFS of four fullerene derivatives, PCBM, bis-PCBM, ICBA, and PC70BM from their PLDMR or photo-EDMR spectra. Figure 6 depicts the in-phase full-field fullerene TE spectra in (99:1) blends with SY-PPV which will be analyzed as a narrow central line superimposed on broader triplet spectra.

For PCBM and bis-PCBM [Figs. 6(a) and 6(b)] two symmetrically positioned maxima with outer pedestals can be recognized as broadened features of the powder spectrum of (close to) axial triplet systems. Indeed, good fits of these spectra were obtained by EPR simulations [33] with the ZFS parameters $(D, E)$ listed in Table I (conventional spin Hamiltonian [23]; see Appendix A), with a significantly smaller $D$ value for the bis- compared to the monoadduct. The broadening of features is ascribed to inhomogeneity of the fullerene surrounding in the polymer matrix leading to a distribution of the values of the ZFS parameters introduced using so-called strain parameters $(\delta D, \delta E$ : FWHM of Gaussian distribution). Note that the $E$-strain values for PCBM and bis-PCBM are much larger than the average values of $E$, pointing to a broad distribution around axial symmetry. The small rhombicity $(3 E / D=0.20)$ and $g$ anisotropy were introduced to reach optimal correspondence among the characteristic features (maxima, shoulders, and extrema) in experiment and simulation. This analysis is further supported by the precise prediction of the position of the half-field transition. 
TABLE I. Zero-field splitting parameters of the studied fullerene derivatives determined from the analysis of the full-field TE spectra presented in Fig. 6 (discussed in Sec. III B). Strain parameters $\delta D$ and $\delta E$ are given for the axial and rhombic ZFS parameters $D$ and $E$ (in megahertz). Comparison is made with the parent molecules $C_{60}[37]$ and $C_{70}[39,40]$ in other matrices and with reported values from the transient EPR of PCBM and PC70BM in frozen solutions [36].

\begin{tabular}{lccccccccrr}
\hline \hline & PCBM & bis-PCBM & $\begin{array}{c}\text { ICBA } \\
\text { This paper }\end{array}$ & PC70BM- $\alpha$ & PC70BM- $\beta$ & PCBM & $\begin{array}{c}\text { PC70BM- } \alpha \\
\text { From transient EPR [36] }\end{array}$ & $\begin{array}{c}\mathrm{C}_{60} \\
{[37]}\end{array}$ & $\begin{array}{c}\mathrm{C}_{70}{ }^{\mathrm{a}} \\
{[40]}\end{array}$ \\
\hline$D^{\mathrm{b}}$ & 300 & 250 & 272 & 359 & 359 & 314 & 256 & 367 & -340 & $147-151$ \\
$\delta D$ & 70 & 70 & 50 & 90 & 70 & & & 12 & 15 \\
$E^{\mathrm{b}}$ & 20 & 20 & 39 & 28 & 0 & 22 & 0 & $22-42$ \\
$\delta E$ & 90 & 90 & 70 & 90 & 70 & & & \\
\hline \hline
\end{tabular}

${ }^{\mathrm{a}}$ Range of values in several matrices.

${ }^{\text {b}}$ Estimated error $\pm 5 \mathrm{MHz}$ on our measured $D$ values and $\pm 3 \mathrm{MHz}$ on $E$ values. Signs of $D$ undetermined except for the parent compounds $[37,39]$.

Moving on to ICBA, more complexity appears in the spectrum with indications of substructures in the outer wings. This can be ascribed to a lower symmetry of the triplet state induced by the two indene adducts. In the spectral simulation shown in Fig. 6(c) a single triplet is introduced, this time with more rhombicity $(3 E / D=0.43$; see parameters in Table I). The pair of maxima close to the central peak as well as the outer steps and their substructures are quite well reproduced. The origin of the remaining discrepancies with the experimental spectrum will be discussed below. ICBA has an additional weak and wider spectrum outside the expected range for the fullerene TE. Similar features have been reported in the earlier studies of PLDMR in $\mathrm{C}_{60}$ and $\mathrm{C}_{70}$ [34], whereas they are not systematically found in other work. We assume that these features arise from residual impurities and are not intrinsically related to the ICBA molecules.

The in-phase photo-EDMR full-field TE spectrum of PC70BM is interestingly different from that of the $C_{60}$ derivatives. It contains two shoulders between the maxima and the outer steps and cannot be fitted as a single full-field spectrum corresponding to one type of triplet. It is known from literature that different isomers of PC70BM exist [35], and two isomers ( $\alpha$ and $\beta$ types, $85 \%$ and $15 \%$ population) were invoked in a recent study [36] involving PC70BM blended with a different polymer to explain similar additional features in transient EPR triplet spectra. In both isomers the adduct is attached onto the same benzene ring nearest to the "pole" of the "bucky baseball" but at adjacent conjugated bonds (at slightly different distances from the longer axis). We presume this variation has little effect on the magnitude of the ZFS but will mainly affect the symmetry of the triplet spin distributions. With this premise, we simulate the full-field TE photo-EDMR spectrum of PC70BM with a combination of two full-field spectra of the same $D$ value, the majority species possessing a moderate rhombic character and the other one axially symmetric. The separate components and the overall fittings using the parameters in Table I are shown in Fig. 6(d). The approach for analysis of this composite spectrum is not unique and different from that followed by Poluektov et al. [36], who came up with two near-to-axial triplet excitations with different $D$ values. Their fitting parameters are also listed for comparison in Table I, and comparable curves are obtained from both sets in simulation of the present spectra in photo-EDMR as well as the reported ones in transient EPR.

In Table I we also included for comparison the literature values of spin-Hamiltonian parameters of the triplet states of the parent fullerene molecules. Interestingly, the values we obtain for the $\mathrm{C}_{60}$ derivatives are quite close to those of the parent molecule [37]. The spontaneous symmetry breaking in the excited state that gives rise to the ZFS for this highly symmetric molecule seems to be still the dominant factor (only limited reduction of the $D$ value by $12 \%, 26 \%$, and 20\% for PCBM, bis-PCBM, and ICBA, respectively), and eventually the adducts mainly define the preferential direction for the (near-) axial relaxation. Comparison can also be made with the ZFS parameters determined by Bortolus et al. [38] from transient EPR for three monoadducts of $\mathrm{C}_{60}$ with $D$ values and rhombicity in the same range as for PCBM. Also for some of the investigated bisadducts they obtained similar ZFS parameters but only for relative positions of the adducts near opposite to each other on the buckyball.

For $\mathrm{C}_{70}$ the situation is very different. Despite the lower ground-state symmetry, the triplet ZFS in the parent compound $[39,40]$ is less than half of that of $\mathrm{C}_{60}$, and still the $D$ value we find for PC70BM is larger than in any of the $\mathrm{C}_{60}$-type molecules. In this case it seems the coupling with the adduct is the dominant factor in the anisotropic properties of the excited electronic state.

Our simulations reproduce well the shape and position of the peaks and shoulders in the different TE spectra, but some discrepancies in relative intensities remain. This is not surprising taking into account that the EPR simulations do not take into account the expected angular dependence in PLDMR or EDMR detection efficiency for which unfortunately no general description is available. However, other factors may be involved as well. Indeed, any of the bisadducts can exist in different isomers. In the case of bis-PCBM it seems from the good fitting with a single triplet spectrum that the differences between isomers are not important and hence not resolved in the spectra. The relative position of the adducts seems to have relatively little influence on the ZFS parameters of the triplet state which are also near to those of the parent $\mathrm{C}_{60}$ molecule [37] (see Table I). For ICBA the deviation from axial symmetry is significant, and there is no reason 
to expect that different isomers will yield the same magnitude or rhombicity of the ZFS. Therefore alternative simulations of the ICBA spectrum should not be excluded in which contributions of different isomers could appear as in the case of PC70BM.

Finally we consider the PLDMR spectra of pristine polymer films which besides the central sharp peak include a very broad full-field spectrum attributed to the polymer TEs. As previously reported for several other PPV compounds, this can be described by a spin Hamiltonian of purely rhombic symmetry, i.e., $3 E / D \cong 1$, with $D=1.42$ and $1.46 \mathrm{GHz}$ for MDMO-PPV and SY-PPV, respectively. The values are similar to other PPV polymers and somewhat smaller than those of several PPV oligomers with $D=1.65-1.71 \mathrm{GHz}[26,41]$ ( $3 E / D \cong 1$ also in the latter cases). In a number of studies of the OMAR effects in the polymer:fullerene blend films both in dark and under photoexcitation, the magnetic-field effects are satisfactorily explained on the basis of interaction of charge excitations in the polymer $[42,43]$. The magnetic-field effects of pristine fullerene devices are generally weak compared to polymeric devices [44]. The relative small ZFS parameters of TEs in fullerene molecules, compared to those in polymers, may result in a lower TE-P scattering yield and hence smaller effects on current or PL. This may be the reason magnetic-field effects of PCBM via the TE-P interaction did not appear to be essential for the modeling of the OMAR effect in SY-PPV:PCBM devices [45].

\section{Spin-dependent triplet exciton-polaron annihilation vs recombination of polarons}

EDMR and PLDMR spectroscopies have previously been applied to investigate spin-dependent interactions in OLEDs and organic solar cells based on the sign of the $g \sim 2$ sharp central EDMR and PLDMR spectra [20]. A positive $g \sim 2$ sharp central EDMR signal has been attributed to enhanced TE-trapped P annihilation [46], which sets a trapped P free and at the same time deexcites the TE to the ground state. A negative EDMR signal is attributed to MW resonant enhancement of $\mathrm{P}$ recombination by equilibrating the population of the spin-up and spin-down states [28,47] or to bipolaron formation [48]. Likewise in PLDMR studies, a positive resonant signal is attributed to reduction in $\mathrm{SE}$ quenching that increases fluorescence [49] or to TE-P annihilation [50]. Negative PLDMR is attributed to enhanced formation of BPs which reduces the radiative recombination rate between Ps [48]. As a result, the sign of the EDMR signal can be dependent on the sample, experimental conditions (e.g., temperature [51]), as well as the MW modulation frequency [24,25]. In some instances, the EDMR spectrum appears to be a superposition of two contributions which have opposite signs [52]. Combining PLDMR and photo-EDMR spectroscopies both on films and on devices at sufficiently slow MW modulation, we are able to elucidate the microscopic spin-dependent processes that contribute to both photocurrent and PL. This is achieved by monitoring the sign of the in-phase and quadrature signals of both the photo-EDMR and the PLDMR spectra in pristine SY-PPV and SY-PPV:fullerene blends as a function of temperature.

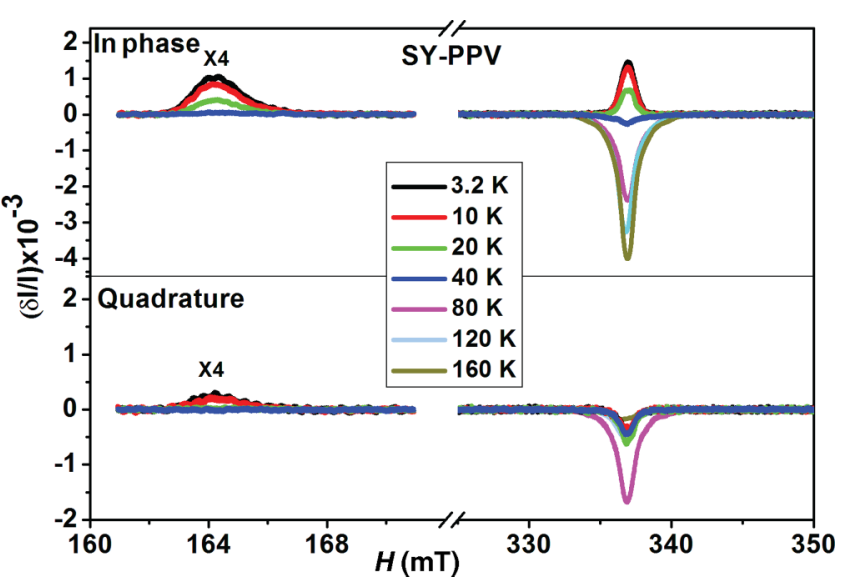

FIG. 7. (Color online) Temperature dependence of the in-phase and quadrature spectra of a pristine SY-PPV diode, showing the sign change in the in-phase signal of the sharp central $g \sim 2$ photo-EDMR peak while the corresponding quadrature signal remains negative in the whole temperature range. The device is photoexcited at $2.71 \mathrm{eV}(\lambda=457 \mathrm{~nm})$. (The sharp central $g \sim 2$ PLDMR spectrum remains positive as a function of temperature; see Appendix B.)

Figure 7 shows both the in-phase and the quadrature $g \sim 2$ sharp central peaks and half-field photo-EDMR TE spectra (see Appendix B for the $g \sim 2$ sharp central PLDMR spectra) in pristine SY-PPV devices as a function of temperature. The in-phase photo-EDMR signal of the $g \sim 2$ sharp central peak changes sign when the temperature is increased. The quadrature signal of the $g \sim 2$ sharp central peak remains negative throughout the studied temperature range. Also, both vector components of the half-field photo-EDMR signal retain their positive signs with temperature. The half-field spectra are detected only at low temperatures, owing to the decreasing lifetime and spin dephasing time of TEs in polymers at higher temperatures. At temperature $T<40 \mathrm{~K}$, the in-phase and quadrature photo-EDMR spectra of the central peak have positive and negative signs, respectively. As the temperature is increased and while the half-field signatures disappear, the in-phase signal of the $g \sim 2$ peak reverses its sign from positive to negative.

At low temperatures, the dominant long-living charge excitations in the polymer are TEs and trapped Ps. The latter are favored by the insufficient thermal activation energy available for $\mathrm{P}$ detrapping and by the slow $\mathrm{P}$ hopping rate [53]. TE-P annihilation process deactivates the TE to the ground state and sets some of the trapped Ps free. A number of these mobile Ps can then combine with negative polarons to SE and may eventually radiatively recombine to the ground state. This recombination decreases the photocurrent by reducing the number of available free Ps. Positive in-phase and negative quadrature photo-EDMR signals indicate that the contribution of TE-P annihilation dominates the $g \sim 2$ in-phase photo-EDMR signal having a faster response to the MW excitation than the $\mathrm{P}$ recombination which dominates the quadrature signal because its response time is comparable to the modulation period. As the temperature is increased the lifetime as well as the spin dephasing time of the TEs shortens, 
and as a result the probabilities for TE-P annihilation as well as for MW-induced transition are decreasing. Meanwhile the population of free polarons increases due to improved thermal activation energy for charge detrapping and higher polaron hopping rate. The sign change in the in-phase signal of the $g \sim 2$ sharp central photo-EDMR spectrum appears when the half-field TE signal disappears, i.e., when the TE-P annihilation is subdued. Consequently, at higher temperatures both the in-phase and the quadrature signals of the photoEDMR spectrum are dominated by the contribution from recombination of distant Ps.

The EDMR detection of the TEs at low temperatures via full-field (or half-field) photo-EDMR spectra can be attributed to TE-P interaction where resonant change in spin population distribution over the triplet spin levels alters the average TE lifetime that consequently changes the yield of TE-P annihilation reaction. If the average TE lifetime increases as a result of MW resonant redistribution of spins among the triplet sublevels, this fosters the detrapping of Ps. The TE-P annihilation also indirectly increases the population of SEs via the combination of Ps into SEs as well as the regeneration of the ground states from the decay of the TEs. This is consistent with the observed temperature-independent positive sign of the in-phase and quadrature signals of the sharp central line in the PLDMR spectrum.

Investigation of spin-dependent processes via sign changes in photo-EDMR and PLDMR spectra in lightly fullerenedoped SY-PPV is more complex due to additional spindependent interaction in the fullerene component. Here, we assume that the scant $\mathrm{CT}$ pair population at molecular contact sites between the blend components has little impact on both the photo-EDMR and the PLDMR signals.

Figure 8 depicts the in-phase and quadrature photo-EDMR spectra of the SY-PPV(99:1)fullerene device at different temperatures. The sign of the half-field photo-EDMR signature of the SY-PPV component in the blend devices is similar to those of the pure SY-PPV diode. As discussed in more detail below, the temperature dependence of the $g \sim 2$ sharp central line can still be understood in the same framework as we considered for the pristine polymer. First we will pay attention to the quite particular behavior of the EDMR signals of the fullerene TEs, which are observable in about the same temperature range as the TEs in SY-PPV. In parallel PLDMR experiments the in-phase and quadrature signals of the spectra in the full-field and half-field regions as well as for $g \sim 2$ sharp central peak were found to be positive in each of the components of the SY-PPV:fullerene blend (see the PLDMR of blends with PCBM and ICBA in Appendix C).

Surprisingly, although both in-phase and quadrature signals of the half-field TE EDMR spectra of PCBM and ICBA are positive, their full-field signals are consistently negative [Figs. 8(a) and 8(b), higher fullerene concentrations presented in Appendix D], both in phase and quadrature. Also in photo-EDMR spectra of SY-PPV:PC70BM devices, both the in-phase and the quadrature signals of the half-field transitions are positive. However, the sign of the in-phase signal of the full-field EDMR spectrum of PC70BM is positive, whereas the weaker quadrature signal has a negative sign. These different
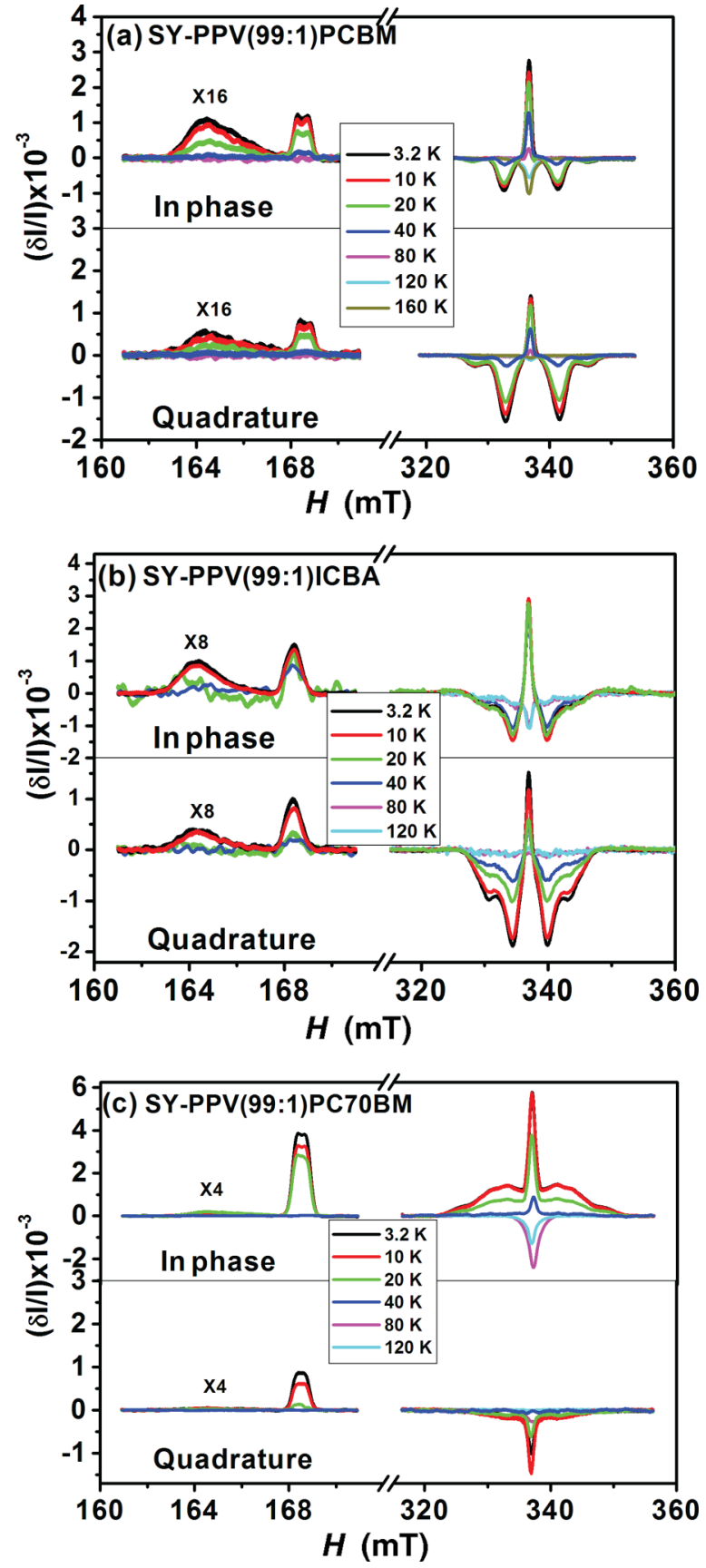

FIG. 8. (Color online) Temperature dependence of the in-phase and quadrature photo-EDMR spectra of (99:1) blends of SY-PPV with (a) PCBM, (b) ICBA, and (c) PC70BM, showing the sign change with temperature of both the in-phase and the quadrature signals of the sharp central $g \sim 2$ peak . The device is photoexcited at $2.71 \mathrm{eV}(\lambda=457 \mathrm{~nm})$. (The corresponding sharp central $g \sim 2$ PLDMR spectra of these blend devices remain positive at all temperatures; see Appendix C for PLDMR spectra and Appendix D for additional photo-EDMR spectra at higher fullerene fractions.)

observations are intriguing and at this point unexplained. The difference in sign between full-field and half-field EDMR signals for the TEs of the $\mathrm{C}_{60}$ derivatives calls for inspection of the possible mechanisms leading to gain and loss of 
photocurrent for these two resonances in the same triplet. Also, the sign inversion for the full-field in-phase signal going from the $\mathrm{C}_{60}$ derivatives to $\mathrm{PC} 70 \mathrm{BM}$ is remarkable. It is interesting to note here that opposite signs have been determined for the $D$ value in the parent molecules $[37,41]$ and thus confirmation of the sign of $D$ for PC70BM would be of interest. For PC70BM there is the additional special situation of opposite signs for in-phase and quadrature signals at full field. As in the case of the $g \sim 2$ central peak in pristine SY-PPV, this seems to indicate the presence of several spin-dependent interactions with different response times. TE-TE annihilation processes were previously invoked in the PLDMR study of PPV oligomers [41] and might contribute here to the full-field photo-EDMR spectra of PC70BM in combination with TE-P annihilation.

The temperature dependence of the in-phase signal of the $g \sim 2$ sharp central photo-EDMR spectrum in SYPPV:fullerene blend devices exhibits the same trends as in pure SY-PPV devices. The in-phase signal changes sign from positive to negative when the TE photo-EDMR (fullor half-field) spectra of either of the material components are disappearing at higher temperatures. Again we ascribe this to competition between the TE-P annihilation and the $\mathrm{P}-\mathrm{P}$ recombination process, dominant at lower and higher temperatures, respectively. However, different situations of relative importance and response times of the two processes seem to occur in the three blends and in pristine SYPPV. Indeed, in blends with $\mathrm{C}_{60}$ derivatives the quadrature signal of the $g \sim 2$ sharp central photo-EDMR spectrum also changes sign as the in-phase signal. Interestingly, the full-field TE spectra of PCBM and ICBA in the blend have stronger quadrature signals at low temperatures, indicating a relatively slow MW resonant response of the TE-P annihilation in the acceptors. When the temperature is increased the effective lifetime of TEs in the acceptors shortens, and around this temperature the MW resonant contribution of the TE-P annihilation to the central line is overtaken by the recombination of polarons. The observations in the PC70BM blend for the $g \sim 2$ central peak are more similar to those in pristine SY-PPV: The quadrature signal is negative for all temperatures pointing to a faster TE-P annihilation as can also be inferred from the weaker quadrature full-field PC70BM signal. The observation of consistently positive in-phase and quadrature signals in the parallel PLDMR measurements for this $g \sim 2$ sharp central line (see Appendix B) is once more consistent with our assignment of spin-dependent contributions of TE-P annihilation and P-P recombination processes.

\section{CONCLUSIONS}

Both PLDMR and EDMR spectroscopies in lightly fullerene-doped MDMO-PPV and SY-PPV reveal that the production of fullerene TEs is dependent on the relative triplet alignment as well as the ET transfer coupling strength between the blend components. In lightly fullerene-doped MDMO-PPV the polymer TEs are the lowest-lying triplet states and are readily detected via their full-field (or half-field) signatures. These TEs are produced as a result of direct ISC from the polymer SEs and possibly by triplet-triplet ET from the fullerene TEs. The observation of a weak full-field TE PLDMR spectrum of ICBA is attributed to a relatively better singlet-singlet ET coupling between MDMO-PPV and ICBA compared to PCBM. In lightly fullerene-doped SY-PPV in which the polymer $E_{\mathrm{T}}$ value is higher than that of the fullerene, intense EDMR and PLDMR signatures of the fullerene TEs are observed. These TEs are produced by direct ISC from the singlet state in the fullerene and eventually by triplet-triplet ET from the polymer triplet level. The singlet state of the fullerene is mainly populated by ET from the polymer due to a stronger singlet-singlet ET coupling.

By judiciously applying EPR spectral simulation to the PLDMR or EDMR full-field spectra, we have determined the ZFS parameters of the TEs on the fullerene derivatives and in pristine SY-PPV. The $D$ values of TEs in the fullerenes are estimated to be approximately four to five times smaller than those of the polymers. The ZFS of TEs in PCBM and bis-PCBM exhibit ZFS with close to axial symmetry, whereas in ICBA the ZFS has a stronger rhombicity. We also resolve the EDMR full-field spectrum of PC70BM into two components, one axial and the other with a stronger rhombic mix, which are attributed to the previously reported $[35,36] \alpha$ - and $\beta$ type isomers of PC70BM. Similar to other PPV compounds, the TE spectra in SY-PPV are well described by a purely rhombic ZFS $(3 E / D \cong 1)$ with a slightly higher $D$ value than in MDMO-PPV.

Finally, we correlate the sign change in both the in-phase signals of the $g \sim 2$ central sharp photo-EDMR signal in the pure as well as in the lightly fullerene-doped SY-PPV device with the disappearance of the TE signal of the polymer or/and the fullerene molecules at increasing temperature. We attribute the change in sign to a transition from TE-P annihilation to a recombination of $\mathrm{P}$-dominated regimes. The former dominates at very low temperatures where the lifetime of TEs is sufficiently long and most of the Ps are frozen out in trap states. However, as the temperature is increased and consequently the lifetime of the TEs become short, the contribution of TE-P annihilation is superseded by P-P recombination which increases as Ps get thermally excitated. This assignment is also consistent with the PLDMR data where both the in-phase and the quadrature signals always retain positive signs with temperature.

In conclusion, we have shown that (photo-) EDMR and PLDMR spectroscopies can complement traditional spectroscopies to unravel the nature of charge excitations and their spin-dependent interactions in polymeric devices. In particular triplet excitons, such as in the fullerene molecules, which are weakly radiative and difficult to identify by conventional spectroscopies, can unambiguously be traced and characterized. This offers perspectives of better understanding and control of triplet excitons which play a key role in the optical efficiency and stability of the state-of-the-art fluorescent and phosphorescent OLEDs. Both EDMR and PLDMR also are becoming invaluable tools to investigate organic photovoltaic devices whose active region is composed of organic materials in layers or blends where conventional techniques often lack the required selectivity. 


\section{ACKNOWLEDGMENTS}

This work was realized with financial support from the fund for Scientific Research-Flanders (FWO) through the Projects No. G.0555.10N and No. G.0888.12N. Also, partial funding was provided by the government agency for Innovation by Science and Technology in Flanders (IWT) via the SBOProject No. 090047 "Silasol." Investment funds for advanced electron-paramagnetic-resonance instrumentation from the Flemish Hercules Foundation via Project No. AUHA013 are kindly acknowledged. This research was also supported by the Dutch Technology Foundation (STW), which is part of the Netherlands Organization for Scientific Research (NWO).

\section{APPENDIX A: ZERO-FIELD SPLITTING PARAMETERS OF TRIPLET SPIN SYSTEMS}

For a weakly interacting two-spin system in the triplet state $(S=1)$, the spin Hamiltonian $H$ is formulated as

$$
\mathcal{H}=\mu_{B} \vec{H} \cdot \stackrel{\leftrightarrow}{g} \cdot \vec{S}+\vec{S} \cdot \stackrel{\leftrightarrow}{D} \cdot \stackrel{\leftrightarrow}{S}
$$

where the first term is the Zeeman energy splitting in the presence of an external field $\vec{H}$ with the matrix $\overleftrightarrow{g}$ describing the (eventually) anisotropic coupling [35]. The second term is the so-called ZFS of dipolar origin in which $\stackrel{\leftrightarrow}{D}$ is a traceless spin-spin coupling tensor of rank 2. Expressed in the framework of principal directions of the $\stackrel{\leftrightarrow}{D}$ tensor, $\mathcal{H}$ is written as

$$
\mathcal{H}=\mu_{B} \vec{H} \cdot \stackrel{\leftrightarrow}{g} \cdot \vec{S}+D\left[S_{Z}^{2}-\frac{1}{3} S(S+1)\right]+E\left(S_{X}^{2}-S_{Y}^{2}\right)
$$

where $D$ and $E$ are now called ZFS parameters and reflect the strength and symmetry of the spin dipolar interaction. In disordered materials, such as studied here, the triplets are expected to have random orientation with respect to $\vec{H}$. Integration over the orientational distribution results in the so-called powder spectrum characteristic for these triplet systems. When the ZFS is small compared to the Zeeman term, variation from axial symmetry $(E=0)$ to the full rhombic case $(3 E / D=1)$ leads to well-known symmetric patterns with specific features that can be used to guide the analysis. The full-field EPR triplet spectrum has singularities at $H=H_{0} \pm(D-3 E) / 2 g \mu_{B}$, shoulders at $H=H_{0} \pm(D+$ $3 E) / 2 g \mu_{B}$, and steps of the outer pedestal at $H=H_{0} \pm$ $D / g \mu_{B}$. The corresponding half-field powder spectrum has a singularity at $H=\frac{H_{0}}{2} \sqrt{1-\left[\frac{4}{3}\left(D^{2}+3 E^{2}\right) /\left(g \mu_{B} H_{0}\right)^{2}\right]}$, where $H_{0}$ is the resonance field position in the absence of the ZFS term.

The features get smoothed in real spectra by different line broadening effects. As discussed in Sec. III B of the main paper, a broad distribution of $D$ and $E$ values exists in the present materials due to variations in the surrounding of the molecules. The EPR simulations incorporate the integration over all orientations as well as over a Gaussian parameter distribution with $\delta D$ and $\delta E$, the FWHM around central values $D$ and $E$ [34].

\section{APPENDIX B: TEMPERATURE DEPENDENCE OF THE $g \sim 2$ SHARP CENTRAL PLDMR SIGNAL IN PRISTINE SY-PPV}

Contrary to the in-phase photo-EDMR (see Fig. 7 in the main paper), no sign change is observed in the $g \sim 2$ sharp central PLDMR spectrum with increasing temperature. Both TE-P annihilation and recombination of polarons enhance the PL output at magnetic-resonance conditions. Films are photoexcited at $2.71 \mathrm{eV}(\lambda=457 \mathrm{~nm})$, close to the absorption peak of the SY-PPV polymer.

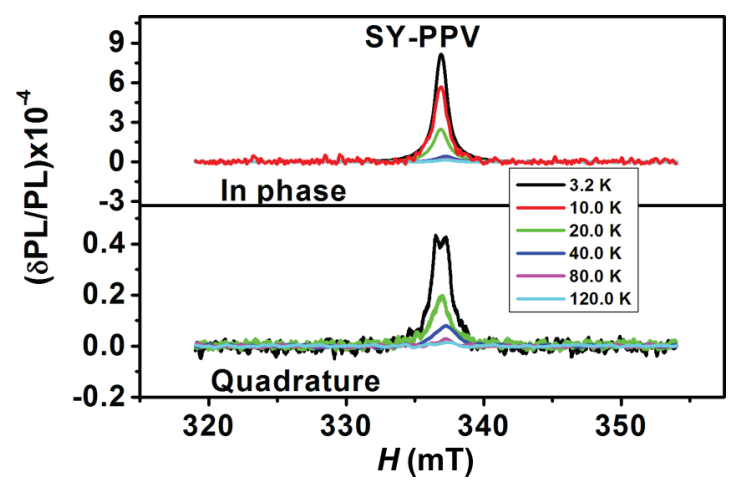

\section{APPENDIX C: TEMPERATURE DEPENDENCE OF PLDMR SPECTRA OF SY-PPV:FULLERENE BLEND FILMS}

The PLDMR signal of: (a) SY-PPV(99:1)PCBM and (b) SY-PPV(99:1)ICBA blend films exhibit consistently positive signals in all parts of the spectrum, TE spectra in both blend components, as well as the $g \sim 2$ sharp central peak. Both blend films are photoexcited at $2.71 \mathrm{eV}(\lambda=457 \mathrm{~nm})$.
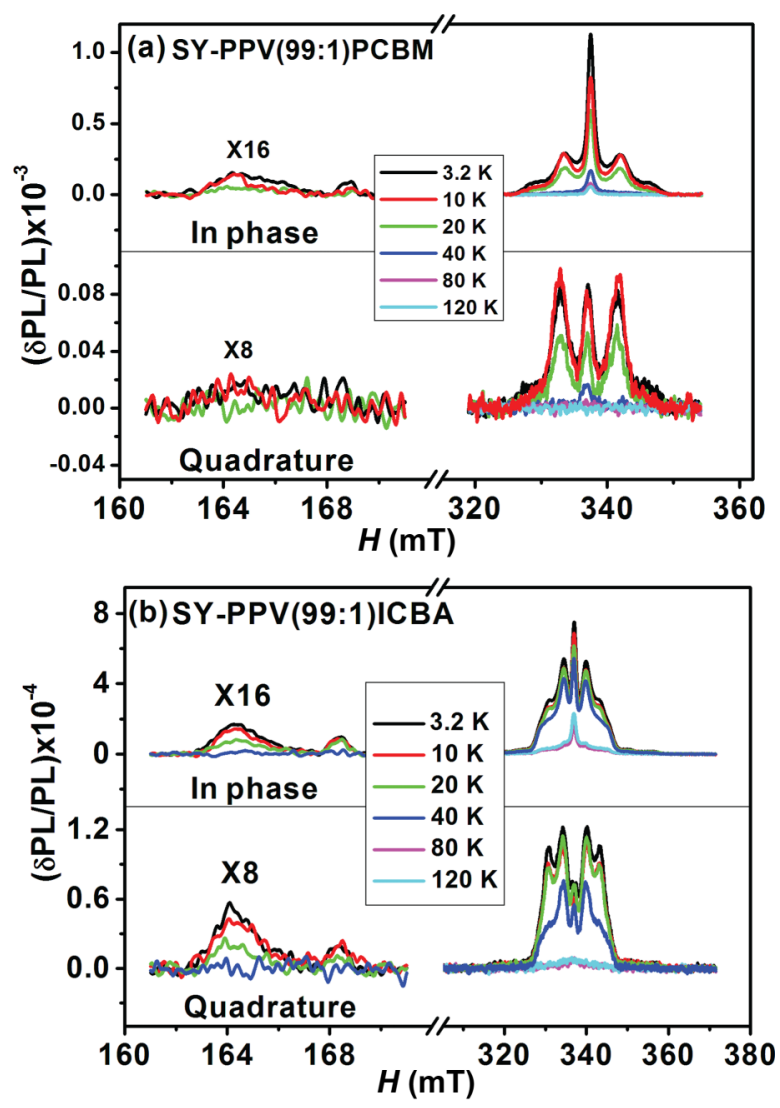


\section{APPENDIX D: PHOTO-EDMR SPECTRA IN SY-PPV: FULLERENE DEVICES OF VARYING FULLERENE FRACTIONS}

The photo-EDMR spectra of: (a) SY-PPV(97:3)PCBM, (b) SY-PPV(90:10)PCBM, and (c) SY-PPV(90:10)PC70BM devices as a function of temperature. Sign changes, both in the in-phase and quadrature signals of the photo-EDMR spectra, are observed for some devices. As discussed in Sec. III C (of the main paper), different situations can occur depending on the relative importance of different contributions and on their response times. In the blends with PCBM and ICBA the contribution of TE-P annihilation to the EDMR signal is dominating the quadrature signal at low temperatures, pointing to a slower TE-P annihilation in these acceptors. Devices are photoexcited with laser energy of $2.71 \mathrm{eV}(\lambda=457 \mathrm{~nm})$.
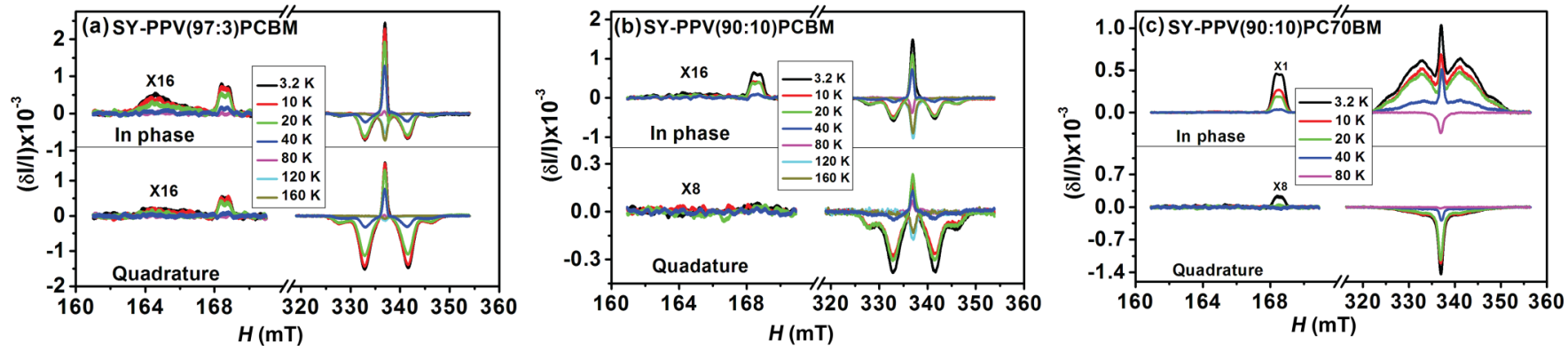

[1] I. A. Howard, J. M. Hodgkiss, X. Zhang, K. R. Kirov, H. A. Bronstein, C. K. Williams, R. H. Friend, S. Westenhoff, and N. C. Greenham, J. Am. Chem. Soc. 132, 328 (2010).

[2] J. M. Hodgkiss, S. Albert-Seifried, A. Rao, A. J. Barker, A. R. Campbell, R. A. Marsh, and R. H. Friend, Adv. Funct. Mater. 22, 1567 (2012).

[3] L. Sudha Devi, M. K. Al-Suti, C. Dosche, M. S. Khan, R. H. Friend, and A. Köhler, Phys. Rev. B 78, 045210 (2008).

[4] T. M. Clarke and J. R. Durrant, Chem. Rev. 110, 6736 (2010).

[5] G. A. H. Wetzelaer, M. Kuik, and P. W. M. Blom, Adv. Energy Mater. 2, 1232 (2012).

[6] J. A. Bartelt, Z. M. Beiley, E. T. Hoke, W. R. Mateker, J. D. Douglas, B. A. Collins, J. R. Tumbleston, K. R. Graham, A. Amassian, H. Ade, J. M. J. Fréchet, M. F. Toney, and M. D. McGehee, Adv. Energy Mater. 3, 364 (2013).

[7] R. A. Street, M. Schoendorf, A. Roy, and J. H. Lee, Phys. Rev. B 81, 205307 (2010).

[8] C. Deibel, A. Wagenpfahl, and V. Dyakonov, Phys. Rev. B 80, 075203 (2009).

[9] R. A. Street, Phys. Rev. B 82, 207302 (2010).

[10] M. Cox, E. H. M. van der Heijden, P. Janssen, and B. Koopmans, Phys. Rev. B 89, 085201 (2014).

[11] A. Kadashchuk, V. I. Arkhipov, C.-H. Kim, J. Shinar, D.-W. Lee, Y.-R. Hong, J.-I. Jin, P. Heremans, and H. Bässler, Phys. Rev. B 76, 235205 (2007).

[12] C. J. Brabec, G. Zerza, G. Cerullo, S. De Silvestri, S. Luzzati, J. C. Hummelen, and S. Sariciftci, Chem. Phys. Lett. 340, 232 (2001).

[13] S. Westenhoff, I. A. Howard, J. M. Hodgkiss, K. R. Kirov, H. A. Bronstein, C. K. Williams, N. C. Greenham, and R. H. Friend, J. Am. Chem. Soc. 130, 13653 (2008).

[14] T. D. Nguyen, Y. Sheng, J. Rybicki, G. Veeraraghavan, and M. Wohlgenannt, Synthetic Metals 160, 320 (2010).

[15] J. Kalinowski, M. Cocchi, D. Virgili, P. Di Marco, and V. Fattori, Chem. Phys. Lett. 380, 710 (2003).

[16] P. A. Bobbert, T. D. Nguyen, F. W. A. van Oost, B. Koopmans, and M. Wohlgenannt, Phys. Rev. Lett. 99, 216801 (2007).
[17] P. Desai, P. Shakya, T. Kreouzis, W. P. Gillin, N. A. Morley, and M. R. J. Gibbs, Phys. Rev. B 75, 094423 (2007).

[18] M. Cox, P. Janssen, F. Zhu, and B. Koopmans, Phys. Rev. B 88, 035202 (2013).

[19] J. Mezyk, R. Tubino, A. Monguzzi, A. Mech, and F. Meinardi, Phys. Rev. Lett. 102, 087404 (2009).

[20] J. Shinar, Laser Photonics Rev. 6, 767 (2012).

[21] J. M. Lupton, D. R. McCamey, and C. Boehme, ChemPhysChem 11, 3040 (2010).

[22] P. A. Lane, J. Shinar, and K. Yoshino, Phys. Rev. B 54, 9308 (1996).

[23] J. A. Weil, J. R. Bolton, and J. E. Wertz, Electron Paramagnetic Resonance: Elementary Theory and Practical Applications (Wiley, New York, 1993), Chap. 6, pp.151-190.

[24] S. Y. Lee, S. Paik, D. R. McCamey, and C. Boehme, Phys. Rev. B 86, 115204 (2012).

[25] C. G. Yang, E. Ehrenfreund, and Z. V. Vardeny, Phys. Rev. Lett. 99, 157401 (2007).

[26] S. Cambré, J. De Ceuster, E. Goovaerts, A. Bouwen, and H. Detert, Appl. Magn. Reson. 31, 343 (2007).

[27] H. D. Burrows, J. S. de Melo, C. Serpa, L. G. Arnaut, A. P. Monkman, I. Hamblett, and S. Navaratnam, J. Chem. Phys. 115, 9601 (2001).

[28] M. C. Scharber, N. A. Schultz, N. S. Sariciftci, and C. J. Brabec, Phys. Rev. B 67, 085202 (2003).

[29] B. Zerai Tedlla, F. Zhu, M. Cox, J. Drijkoningen, J. Manca, B. Koopmans, and E. Goovaerts, Adv. Energy Mater. 5 (2015), doi:10.1002/aenm.201401109.

[30] B. P. Rand, S. Schols, D. Cheyns, H. Gommans, C. Girotto, J. Genoe, P. Heremans, and J. Poortmans, Org. Electron. 10, 1015 (2009).

[31] D. Veldman, S. C. J. Meskers, and R. A. J. Janssen, Adv. Funct. Mater. 19, 1939 (2009).

[32] D. Di Nuzzo, G.-J. A. H. Wetzelaer, R. K. M. Bouwer, V. S. Gevaerts, S. C. J. Meskers, J. C. Hummelen, P. W. M. Blom, and R. A. J. Janssen, Adv. Energy Mater. 3, 85 (2013). 
[33] EPR simulations performed with the program EASYSPIN, S. Stoll and A. Schweiger, J. Magn. Reson. 178, 42 (2006); http://www.easyspin.org

[34] P. A. Lane and J. Shinar, Phys. Rev. B 51, 10028 (1995).

[35] M. M. Wienk, J. M. Kroon, W. J. H. Verhees, J. Knol, J. C. Hummelen, P. A. van Hal, and R. A. J. Janssen, Angew. Chem. Int. Ed. 42, 3371 (2003).

[36] O. G. Poluektov, J. Niklas, K. L. Mardis, S. Beaupré, M. Leclerc, C. Villegas, S. Erten-Ela, J. L. Delgado, N. Martín, A. Sperlich, and V. Dyakonov, Adv. Energy Mater. 4, 1301517 (2014).

[37] G. J. B. Van den Berg, D. J. van den Heuvel, O. G. Poluektov, I. Holleman, G. Meijer, and E. J. J. Groenen, J. Magn. Reson. 131, 39 (1998).

[38] M. Bortolus, M. Prato, J. van Tol, and A. L. Maniero, Chem. Phys. Lett. 398, 228 (2004).

[39] X. L. R. Dauw, O. G. Poluektov, J. B. M. Warntjes, M. V. Bronsveld, and E. J. J. Groenen, J. Phys. Chem. A 102, 3078 (1998).

[40] M. N. Uvarov, L. V. Kulik, and S. A. Dzuba, Appl. Magn. Reson. 40, 489 (2011), and references therein.

[41] J. De Ceuster, E. Goovaerts, A. Bouwen, and V. Dyakonov, Phys. Rev B 68, 125202 (2003).

[42] Z. Xu and B. Hu, Adv. Funct. Mater. 18, 2611 (2008).
[43] P. Janssen, M. Cox, S. H. W. Wouters, M. Kemerink, M. M. Wienk, and B. Koopmans, Nat. Commun. 4, 2286 (2013).

[44] F. J. Wang, H. Bässler, and Z. Valy Vardeny, Phys. Rev. Lett. 101, 236805 (2008).

[45] P. Janssen, S. H. W. Wouters, M. Cox, and B. Koopmans, Org. Electron. 15, 743 (2014).

[46] T. D. Pawlik, M. E. Kondakova, D. J. Giesen, J. C. Deaton, and D. Y. Kondakov, J. Soc. Inf. Disp. 17, 279 (2009).

[47] V. Dyakonov, N. Gauss, G. Rösler, S. Karg, W. Riess, and M. Schwoerer, Chem. Phys. 189, 687 (1994).

[48] L. S. Swanson, J. Shinar, A. R. Brown, D. D. C. Bradley, R. H. Friend, P. L. Burn, A. Kraft, and A. B. Holmes, Phys. Rev. B 46, 15072 (1992).

[49] W. Graupner, J. Partee, J. Shinar, G. Leising, and U. Scherf, Phys. Rev. Lett. 77, 2033 (1996).

[50] M. Segal, M. A. Baldo, M. K. Lee, J. Shinar, and Z. G. Soos, Phys. Rev. B 71, 245201 (2005).

[51] G. Li, C. H. Kim, P. A. Lane, and J. Shinar, Phys. Rev. B 69, 165311 (2004).

[52] N. C. Greenham, J. Shinar, J. Partee, P. A. Lane, O. Amir, F. Lu, and R. H. Friend, Phys. Rev. B 53, 13528 (1996).

[53] I. I. Fishchuk, A. Kadashchuk, S. T. Hoffmann, S. Athanasopoulos, J. Genoe, H. Bassler, and A. Kohler, Phys. Rev. B 88, 125202 (2013). 